doi: https://doi.org/ 10.5377/paradigma.v28i46.12838

\title{
Perfil de Ingreso y Trayectoria Académica de los Estudiantes de la Cohorte 2017 de la Universidad Pedagógica Nacional Francisco Morazán
}

\section{Admission Profile and Students' Academic Trajectory of the 2017 Cohort of the Francisco Morazán National Pedagogical University}

\author{
Leví Astul Castro Ordónez ${ }^{l}$ \\ Esther Fonseca Aguilar ${ }^{2}$
}

\begin{abstract}
Resumen
En este estudio se analiza, mediante técnicas descriptivas y multivariadas, el perfil de ingreso y la trayectoria académica de los estudiantes de la cohorte 2017 de la Universidad Pedagógica Nacional Francisco Morazán; para lo cual se consideran la condición demográfica, económica, antecedentes académicos, resultados del test de aptitud académica e indicadores de la trayectoria académica (matrícula, abandono, movilidad, regularidad y éxito académico). Los resultados muestran que un número reducido de variables del perfil de ingreso explica la trayectoria académica, y que la influencia de las variables difiere por año académico analizado y por facultad a la cual están adscritos los estudiantes. También, se encontró que el test de aptitud académica, es predictor del desempeño en los cursos generales de Español y Matemáticas; sin embargo, no aparece regularmente como predictor para otras variables de trayectoria académica. Finalmente, las trayectorias académicas de los estudiantes son bastante irregulares, siendo los desempeños del estudiante durante un año, fuertes predictores del éxito en el año siguiente.
\end{abstract}

Palabras claves: éxito académico, test de admisión, indicadores, regresión logística.

\footnotetext{
${ }^{1}$ 1castro@upnfm.edu.hn. Universidad Pedagógica Nacional Francisco Morazán. Tegucigalpa, Honduras. https://orcid.org/00000001-8839-6879

2 efonseca@upnfm.edu.hn. Universidad Pedagógica Nacional Francisco Morazán. Tegucigalpa, Honduras. https://orcid.org/00000002-0356-9409

Recibido 27 de septiembre de 2021 / Aceptado 22 de noviembre de 2021
} 


\begin{abstract}
This study analyses the admission profile and students' academic trajectory of the 2017 cohort at the Francisco Morazán National Pedagogical University, using descriptive and multivariate techniques; for which the demographic and economic condition, academic background, results of the academic aptitude test and indicators of the academic trajectory (enrollment, dropout, mobility, regularity and academic success) are considered. The study shows that a small number of variables of the admission profile explain the academic trajectory and the influence of the variables differs by academic year analyzed and by academic faculty to which the students are assigned. Furthermore, it was found that the academic aptitude test predicts performance in Spanish and Mathematics courses; however, it does not appear regularly as a predictor for other variables of academic trajectory. Finally, the student's academic trajectories are quite irregular, being student's performances for one year being strong predictors of success in the next year.
\end{abstract}

Keywords: academic success, admission test, indicators, logistic regression.

\title{
Introducción
}

De acuerdo con Lago, et al. (2014), gran parte de los estudios sobre calidad de la educación superior se realizan a partir de 1999 luego del tratado de Bolonia, el cual tuvo un gran impacto en Europa y Latinoamérica. Afirman estos autores que la gran mayoría de los estudios se pueden vincular al estudio del impacto de los procesos de autoevaluación y acreditación, a la comparación de los diferentes sistemas de aseguramiento de la calidad y al análisis de los modelos de evaluación de la calidad de los programas académicos. Quizá uno de los elementos promovidos en este tratado que más ha influido en el estudio de la calidad de la educación superior sea "la adopción de un sistema de titulaciones universitarias fácilmente comparable en toda Europa lo que daba apertura a la movilidad de estudiantes y profesores investigadores por las universidades europeas" (Lago, et al., 2014, p. 159); esto es así, porque obliga a establecer criterios o indicadores que aseguren la calidad como garantía de comparabilidad y movilidad.

Si bien es posible definir una gran cantidad de indicadores de calidad para la educación superior (Buela-Casal et al., 2009; Velásquez de Zapata, 2013), uno de los indicadores sobre los cuales se presta mucha atención es la eficiencia; la cual, en general, se puede definir como la relación entre los recursos invertidos y el producto logrado (López Suárez, et al., 2008). Uno de los aspectos de la eficiencia que presenta serias dificultades para ser estudiado, pero que resulta de vital importancia para las universidades, es la eficiencia terminal; la cual se puede definir como la relación entre el 
número de estudiantes que se inscriben por primera vez a una carrera $\mathrm{y}$, de estos, los que logran egresar en el tiempo estipulado en el plan de dicha carrera (Zandomeni y Canale, 2010).

Hablando de la educación formal, en general, y de las carreras universitarias, en particular, el concepto de eficiencia terminal implica dos momentos: ingreso y egreso. El recorrido entre estos dos puntos está determinado de manera teórica en los planes de las carreras y es lo que se conoce como trayectoria académica teórica. No obstante, vale aclarar que el concepto de trayectoria académica, de acuerdo con Zandomeni y Canale (2010), se puede identificar en diversos estudios como sinónimo de trayectoria escolar y trayectoria educativa. A pesar de ello, tomando en cuenta las consideraciones de estos autores, en esta investigación se utilizará el término trayectoria académica y se definirá como el recorrido de un estudiante desde el ingreso hasta la culminación de los estudios en la educación formal.

La trayectoria académica, como recorrido teórico, si bien es predecible desde el plan de la carrera, no necesariamente es lineal; de tal modo que, si comparamos dos estudiantes exitosos que transitan el plan, apegados al recorrido teórico, podrían seguir rutas diferentes. Por otra parte, la mayoría de los estudiantes no siguen este recorrido teórico. Esto es así, ya que, a pesar de los esfuerzos que puedan realizar las universidades para lograr, por ejemplo, por medio de la asesoría académica, que los estudiantes se apeguen al recorrido teórico, la realidad es que existen muchas variables que determinan el recorrido real; algunas de estas variables no son controlables ni por los estudiantes, ni mucho menos por las universidades.

Zandomeni y Canale (2010) reportan que las universidades realizan estudios de trayectorias académicas con el objetivo de obtener información sistemática para definir estrategias tempranas orientadas a la reducción del rezago académico, el cual se manifiesta a través de variables como abandono, reprobación, repitencia, ausentismo, etc. Estos estudios sobre trayectorias académicas, y quizá debido al creciente uso de test de admisión a la educación superior, pueden incluir la relación entre los puntajes obtenidos en los test de admisión y el éxito académico a lo largo de la carrera universitaria. Vale destacar que el estudio de este tipo de relación también es parte de lo que se conoce como validez predictiva, esencial para la determinación de la calidad de un test; de ahí que la consecuencia lógica del uso creciente de los test implique un incremento en el estudio de este tipo de relaciones. 
Además de estudiar la relación entre los test de admisión y las trayectorias académicas, es común analizar los perfiles de ingreso y su relación con el éxito académico o la trayectoria académica. Esto es así, porque existe evidencia de que algunas variables de los estudiantes (socioeconómicas, de antecedentes académicos, de capital cultural, demográficas, entre otras) explican estas variables de eficiencia. Por ejemplo, Olarte Moyano (2020, p. 34) encontró que el riesgo de desertar es 50\% más en los estudiantes cuya edad está en el primer cuartil respecto a los que tienen otra edad $(\mathrm{p}=0.000)$ y que el riesgo de desertar de los que se encuentran en los estratos sociales más bajos (1 y 2, de acuerdo a su categorización) es $25 \%$ más de los que se encuentran en estratos superiores $(\mathrm{p}=0.002)$. Corominas $(2001, \mathrm{p}$. 138) reporta una mayor frecuencia de cambio en los estudiantes más jóvenes y en aquellos cuyos estudios son financiados por los padres, mientras que el abandono ocurre con mayor frecuencia en los estudiantes mayores y en los que financian sus propios estudios $(\mathrm{p}=0.000)$. En Colombia, el Ministerio de Educación Nacional (2008) encontró como variables explicativas relevantes de la deserción en educación superior el sexo, la edad, estar trabajando al momento del ingreso, el número de hermanos en el hogar, el nivel educativo de la madre, entre otras ( $\mathrm{p} \leq 0.009$ en todos los casos).

En el contexto hondureño, se identifican algunos estudios acerca de la calidad de la educación superior en los cuales se analizan, entre otros, la eficiencia terminal, la matrícula, el rendimiento y la repitencia; sin embargo, estos estudios son principalmente descriptivos y suelen reportar indicadores en términos de porcentajes (Universidad Nacional Autónoma de Honduras [UNAH], 2018; Berríos, et al., 2016; Castillo y Martínez, 2016; Pérez, et al., 2011; Arita, 2010). No obstante, se identificó un estudio en el cual se analiza el rendimiento académico de los estudiantes de la Universidad Zamorano por medio de un modelo de regresión múltiple (Báez, 2013).

En el presente estudio, se analizan las variables que caracterizan el perfil de ingreso y los indicadores de la trayectoria académica de los estudiantes de la cohorte 2017 de las licenciaturas ofertadas por las facultades de Humanidades y de Ciencia y Tecnología de la Universidad Pedagógica Nacional Francisco Morazán (UPNFM), que se imparten tanto en la modalidad presencial como a distancia. El interés de estudiar esta cohorte radica en el hecho que, de acuerdo con el programa oficial, estos estudiantes debieron graduarse en el año 2020 y, además, es la última cohorte en que la demanda superó significativamente a la oferta de la UPNFM. 
Esta investigación constituye una importante contribución a la comprensión de las trayectorias académicas, debido a que se analiza el perfil de ingreso y su relación con diversos indicadores que la explican. Además, a nivel metodológico, es un importante aporte para el análisis de los indicadores de la calidad de la educación superior, ya que se utilizan técnicas multivariadas, como el análisis de clúster y la regresión logística; poco utilizadas en los estudios nacionales consultados.

\section{Discusión Teórica}

Cuando el número de aspirantes supera la oferta de nuevos cupos, las universidades se ven obligadas a definir mecanismos de selección. Estos mecanismos deben cumplir con estándares de calidad que aseguren el acceso a los aspirantes idóneos en términos de los perfiles de ingreso. Entre los recursos que se suelen incluir en estos procesos están los test aptitudinales, los cuales permiten ubicar a los aspirantes en carreras específicas en términos de sus puntuaciones en los test y los criterios de selección (Chain Revuelta, et al., 2003). Sin embargo, esta no es la única razón por la que los centros de educación superior realizan procesos de admisión; algunas lo hacen porque desean captar a los mejores aspirantes o a los que cumplen con un perfil muy específico, como en el caso de la selección de los candidatos a estudiar una carrera docente en Finlandia (Pérez Granados, 2014).

\section{Los Test de Admisión en Honduras}

El interés por medir diferencias individuales y capacidades cognitivas se puede remontar a los trabajos de Galton a finales del siglo XIX y los de Binet a inicios del siglo XX. Otros autores realizaron importantes aportes, entre los que destaca Spearman, quien a inicios del siglo XX propuso un modelo matemático para tratar el error de medida de las puntuaciones de un test. Desde entonces, el uso de test ha ido en aumento al tiempo que se desarrollan y perfeccionan las teorías de medición (Abad, et al., 2011). Los test de aptitud académica, ampliamente utilizados en los procesos de admisión universitaria, se fundamentan en alguna de estas teorías de medición.

Las pruebas de admisión universitaria en Honduras se han utilizado desde la segunda mitad del siglo pasado, pero en pocos centros de educación superior y con muy poco impacto en la cultura de evaluación estandarizada. A inicios de la década del 90 del siglo pasado el Consejo de Educación Superior establece las normas académicas de la educación superior en Honduras; estableciendo, en los artículos 90 al 94, normas específicas de admisión a las universidades, desde su definición hasta su establecimiento como requisito de ingreso (UNAH, 1992). 
En el año 2002, la UPNFM aplica por primera vez un test de aptitud académica ${ }^{3}$, con fines de selección. La función de este test era identificar a los estudiantes más idóneos para realizar estudios universitarios en esta casa de estudio. Desde sus inicios la UPNFM apuntó a desarrollar y administrar su propio test, aprovechando la experiencia acumulada por medio de la Unidad Externa de Medición de la Calidad de la Educación en el diseño, desarrollo y administración de evaluaciones estandarizadas a gran escala, utilizando estándares internacionales y las teorías psicométricas de mayor uso a la fecha (Coordinación de Examen de Admisión [CEA], 2019). La antigua Escuela Nacional de Ciencias Forestales ${ }^{4}$, incluía en el proceso de admisión pruebas de conocimientos generales en matemática, química y biología y un test de aptitud y razonamiento verbal (Solórzano, 2005). Por otra parte, otras universidades del país contratan los servicios del College Board de Puerto Rico, a través de la dirección del Sistema de Admisión de la Universidad Nacional Autónoma de Honduras, para hacer uso de la prueba de aptitud académica.

\section{Relación entre el Perfil de Ingreso y el Desempeño Académico Universitario}

Con relación al perfil de ingreso, según refiere Corominas (2001), en las teorías de Tinto, una de las ideas destacables es que los estudiantes que ingresan a los estudios universitarios lo hacen con un conjunto de características, antecedentes personales, expectativas e intenciones que definen su integración académica y social; características que influyen en la decisión de permanecer o salir de los estudios. La integración académica tiene que ver con el grado de congruencia entre el desarrollo intelectual del estudiante y el clima intelectual de la institución. Los primeros dos años en el nivel superior, son clave en la vida universitaria en lo que respecta a la integración estudiantil (Pérez Alcántara, 2017).

En tal sentido, existe evidencia de que el desempeño previo de los estudiantes en la enseñanza media tiene un poder predictivo respecto al desempeño en los estudios universitarios. Al parecer los estudiantes con buen desempeño académico en educación media, suelen tener mejores métodos de estudio, cierta disciplina y responsabilidad individual, características que garantizan el éxito estudiantil (Soria-Barreto y Zuñiga-Jara, 2014; Chain Revuelta, et al., 2003; Rodríguez Garcés y Padilla Fuentes, 2016). El desempeño académico de educación media tiene un poder predictivo creciente o constante en el desempeño académico en el nivel superior; sin embargo, la correlación de las pruebas de admisión con el desempeño académico tiende a bajar al avanzar en los estudios, es decir estas pruebas tendrían un poder predictivo decreciente (Fischer y Repetto, 2003).

${ }^{3}$ La Universidad Pedagógica Nacional, tiene sus raíces en la otrora Escuela Superior del Profesorado, la cual en sus primeros años aplicaba pruebas de conocimiento a los candidatos para realizar estudios de profesorado; sin embargo, eventualmente dejó de administrar estas pruebas como requisito de admisión.

${ }^{4}$ Hoy Universidad Nacional de Ciencias Forestales, la cual aplica la prueba de aptitud académica del College Board. 
Aunque algunas universidades de la región optan por incorporar información de las calificaciones de los estudios de educación media como variable para la selección de los aspirantes (Rodríguez Garcés y Padilla Fuentes, 2016; Fischer y Repetto, 2003), la UPNFM no las incorpora en el proceso ya que, como se revela en el estudio realizado por la Unidad Medición de la Calidad de la Educación (2017, p. 27-29), estás son poco útiles para discriminar entre estudiantes de acuerdo a su aprovechamiento en los estudios preuniversitarios. Adicionalmente, como afirman Fischer y Repetto (2003, p. 247) "las notas en la educación media tienen distintos significados que dependen del establecimiento educacional: un buen estudiante en un colegio selectivo puede obtener notas peores que un estudiante peor en colegio menos exigente".

\section{La Trayectoria Académica}

Al ingresar a la universidad, los estudiantes inician un proceso de transición del nivel medio al nivel superior, el cual puede ser un proceso desafiante debido, entre otras, a las características sociales, económicas y condiciones de aprendizajes, tal como afirma Corominas $(2001$, p. 127) “A1 final del primer año de universidad es cuando se completa la transición entre secundaria y universidad. Los casos de abandono o cambio (de estudios, de universidad) entre los estudiantes universitarios se dan principalmente en este primer año", y amplía Pérez (2017, p. 23) "El problema de la deserción escolar es multifactorial y están involucrados aspectos de tipo personal, (indisciplina, insatisfacción con la carrera elegida, reprobación), así como de tipo familiar, económico y cambios de lugar de residencia entre otros".

El estudio de esta dinámica en cuanto al recorrido del estudiante desde su ingreso a la universidad hasta el egreso corresponde al estudio de la trayectoria académica, siendo este último un concepto complejo debido a las múltiples dimensiones que determinan su comportamiento. Se requiere estudiar la interacción de experiencias sociales y curriculares, situadas en un tiempo y en un espacio, mediante un abordaje comprensivo del recorrido de los estudiantes durante la vida universitaria (Guevara y Belelli, 2013; Pérez Alcántara, 2017).

El concepto de trayectoria académica está estrechamente vinculado al de eficiencia terminal, el cual es considerado como el indicador más importante en las evaluaciones institucionales y que está estrechamente relacionada con la deserción y el rezago; los cuales afectan el recorrido de las trayectorias teóricas (García Robelo y Barrón Tirado, 2011). Ahora bien, los estudios de las trayectorias académicas son relevantes pues permiten identificar las causas de los recorridos reales vinculadas al bajo rendimiento escolar, la discontinuidad o rezago académico y la reprobación (Pérez Alcántara, 2017). 


\section{El Éxito Académico}

Un concepto vinculado al de trayectoria teórica es el de éxito académico, el cual es el resultado de múltiples factores que se relacionan e interactúan de diversas maneras (Chain Revuelta, et al., 2003). El éxito puede conceptualizarse como el grado de cercanía entre la trayectoria real y la trayectoria teórica (cuanto más próximos, mayor éxito), no obstante, vale destacar que esta forma de concebirlo desestima la realidad de los estudiantes quienes, en términos de sus condiciones, pueden considerar como exitosa su trayectoria tomando en cuenta sus objetivos individuales, más cercanos a su realidad, y no necesariamente a los objetivos institucionales en cuanto a eficiencia terminal.

\section{Métodos y Materiales}

El presente es un estudio cuantitativo con diseño no experimental, correlacional y multivariado. En cuanto al enfoque metodológico para el estudio de la trayectoria académica, en este estudio siguen, principalmente, las recomendaciones de Zandomeni y Canale (2010, p. 62-65), quienes argumentan a favor de un abordaje descriptivo y otro explicativo, ya que estos, en conjunto, permitan una mejor configuración de dichas trayectorias académicas.

\section{Población y Muestra}

La población consta de 4,882 aspirantes que resultaron preseleccionados para iniciar estudios en el primer período académico de 2017 en las carreras que pertenecen a las facultades de Humanidades y de Ciencia y Tecnología de la UPNFM, de los sistemas presencial y a distancia, de la sede central y ocho de los centros regionales a nivel nacional.

De los estudiantes de la población se excluyeron a los preseleccionados en convocatoria complementaria, pues estos no llenaron la ficha de inscripción. También se excluyeron los estudiantes que, habiendo sido preseleccionados, ya se encontraban registrados como estudiantes de la universidad en algún programa de profesionalización. Asimismo, se excluyeron los preseleccionados para el programa de Formación Inicial de Docentes, debido a la naturaleza especial del mismo. Por lo anterior, la muestra está constituida por 3,072 estudiantes.

\section{Fuente de Datos}

La CEA administra, a los aspirantes que participan en el proceso de admisión, una ficha de inscripción que consiste en un cuestionario en línea, en la cual se indaga, entre otras, acerca de variables socioeconómicas, demográficas y de antecedentes académicos. Los datos obtenidos mediante este instrumento son utilizados en este estudio para la selección de variables predictoras, las cuales contribuyen para la construcción del perfil de ingreso; a estos datos nos referimos como FICHA. 
Asimismo, la CEA administra una batería de seis test para las áreas de español, matemáticas, razonamiento y vocación. Los ítems del test de vocación son tipo Likert, mientras que para el resto de test los ítems son de selección múltiple con respuesta única. Las puntuaciones de los aspirantes se estimaron en el marco de la teoría de respuesta al ítem con un modelo de dos parámetros reescalando las puntuaciones con una media de 500 puntos y una desviación típica de 100. Con esta fuente de datos se obtuvo información de los resultados logrados por los aspirantes en tres escalas: llamamos ESPA a la escala que mide el área de español, MATE a la escala que mide el área de matemáticas y RAAB a la escala que mide razonamiento abstracto.

Finalmente, de la base de datos de estudiantes de la Oficina de Registro, la Dirección de Tecnologías de Información proporcionó varios archivos conteniendo datos acerca de la muestra en cuanto a matrícula, aprobación, reprobación, calificaciones, retiros, traslados, entre otros, para los años 2017, 2018 y 2019; a este conjunto de archivos llamamos HISTORIAL. Del estudio se excluyeron los datos de 2020 debido a lo atípico del año académico a causa de la crisis sanitaria por coronavirus.

\section{Definición de Variables}

Del archivo FICHA se obtienen las variables demográficas (sexo, edad, estado civil y grupo poblacional), de antecedentes académicos (tipo de centro según administración, título de educación media, modalidad del centro educativo, año de graduación, está realizando estudios universitarios, ha realizado estudios universitarios), económicas (ingreso familiar promedio, estatus laboral, dependientes, posee vivienda la familia, dependencia económica), y de perfil de admisión (puntaje en ESPA, MATE, RAAB y opción de carrera con la que fue preseleccionado). A partir del archivo HISTORIAL, se tomaron o construyeron las variables relacionadas con la trayectoria académica: matrícula, movilidad, abandono, permanencia, repitencia, aprobación, rendimiento y éxito académico. Vale destacar que estas variables fueron transformadas en función del tipo de análisis realizado; por ejemplo, para los modelos de regresión logística todas las variables predictoras fueron dicotomizadas.

Debido a su relevancia en el proceso de formación de los estudiantes, se decidió estudiar la relación entre los puntajes en el test de admisión y el rendimiento en los espacios pedagógicos Español (FFM1301) y Matemáticas (FFE0301). Estos espacios sientan las bases de formación para adquirir nuevos conocimientos, independientemente de la licenciatura a la que está adscrito el estudiante. Ambos aparecen en el primer año según las mallas curriculares, y aunque algunos 
estudiantes los cursan en períodos posteriores, la mayoría de ellos los toman en el primer periodo académico. Como medida de rendimiento en estos espacios se tomó la calificación obtenida la primera vez que tomaron el curso.

Se definió el éxito académico en el año como "aprobar 12 o más espacios pedagógicos en ese año". Para esta definición, se consideró el hecho que el total de espacios pedagógicos por año en las licenciaturas, de acuerdo con las mallas curriculares, oscila entre 12 y 15 . Por otra parte, el porcentaje de estudiantes que cursa 15 espacios pedagógicos por año es bastante bajo y las comparaciones o construcción de modelos no se pueden justificar.

\section{Análisis de Datos}

Para el análisis, primero se realizó una revisión de cada archivo obtenido a fin de determinar la calidad de cada variable y descartar aquellas con demasiados valores perdidos o con datos inconsistentes. Luego, se procedió a recodificar variables o a crear nuevas a partir de las existentes, según las necesidades de análisis. Se realizó una serie de análisis de distribuciones de casos mediante tablas de frecuencias, tablas de contingencia, gráficos de dispersión, etc., con el fin de comprender las distribuciones de datos y posibles relaciones.

Con el propósito de clasificar a los estudiantes según su puntaje en las escalas ESPA, MATE y RAAB se realizó, para cada una, análisis de conglomerados de k-medias con tres conglomerados y el método iterar y clasificar, solicitando como estadísticos las tablas ANOVA (ANalysis Of VAriance; en español, Análisis de Varianza).

Para determinar la relación entre el test de admisión y desempeño en FFM1301 y FFE0301, se compararon las medias de las calificaciones en estos dos espacios pedagógicos de los estudiantes en cada conglomerado para ESPA, MATE y RAAB, mediante ANOVA, solicitando un contraste post hoc.

Para el estudio de la trayectoria académica, se construyó un diagrama en el cual se muestra el porcentaje de estudiantes activos por año académico separándolas en dos rutas a partir de 2017: los que matricularon 12 o más espacios pedagógicos y los que matricularon menos. A partir de allí, se analizaron los porcentajes de estudiantes que aprobaron todos los espacios pedagógicos, los que reprobaron al menos un espacio pedagógico, los que abandonaron al menos uno y los que no se presentaron en al menos un espacio pedagógico. 
Luego se analizaron algunos indicadores de trayectoria académica, tratando de identificar variables del perfil de ingreso que pudieran explicarlos. Se realizó, para cada escala (ESPA, MATE, RAAB), una prueba de independencia entre las variables "clúster al que pertenece" y "abandono inmediato". Para el abandono después de tres años, se construyó un modelo de regresión logística con las variables de perfil de ingreso y algunas de trayectoria como predictores. Se estudió la movilidad mediante un modelo de regresión logística. También se realizó una prueba de independencia entre las variables cambio de carrera y opción de carrera por la que fue preseleccionado. Se compararon algunos indicadores de trayectoria académica entre estudiantes regulares e irregulares mediante comparaciones de medias para grupos independientes.

Para predecir el éxito académico en cada año (aprobar doce o más espacios pedagógicos), se construyeron modelos de regresión logística con el método Hacia atrás: Wald, con 0.05 como probabilidades para entrar o ser eliminado en el método por pasos; un total de seis modelos fueron construidos, uno por cada año y facultad. Para la selección de las variables predictoras se tomaron en cuenta estadísticos de bondad de ajuste, especificidad, sensibilidad y la interpretabilidad.

\section{Resultados}

En esta sección se presentan los principales resultados de la investigación. Primero se presentan algunos descriptivos para caracterizar la muestra, luego se analiza la relación entre el test de admisión y el rendimiento en FFE0301 y FFM1301. A continuación, se analizan aspectos de la trayectoria académica real y algunos de indicadores como matrícula, abandono, movilidad y rendimiento. Finalmente, se analiza el éxito académico para cada año por facultad.

\section{Características de la Muestra}

De los 3,072 estudiantes en la muestra, 1,095 (35.6\%) fueron asignados a un programa llamado Estudios de Fundamento General y Pedagógicos (EFGP), para estos estudiantes no es posible determinar ni la carrera ni la facultad a la cual estaban adscritos pues la asignación se realizaría después de aprobadas cierto número de espacios pedagógicos generales y de fundamentos pedagógicos. En la Tabla 1 se muestra la distribución de estudiantes por centro universitario de estudio, modalidad y programa al que ingresó. 


\section{Tabla 1}

Estudiantes por centro de estudio, programa de ingreso y modalidad de estudio.

\begin{tabular}{ccccc}
\hline Centro de estudio & \multicolumn{2}{c}{ EFGP } & $\begin{array}{c}\text { Regular } \\
\text { Presencial }\end{array}$ & Total \\
\hline Choluteca & 151 & Presencial & 24 & 175 \\
Gracias & 102 & 18 & & 120 \\
Juticalpa & & 58 & & 58 \\
La Ceiba & 219 & & 158 & 377 \\
Nacaome & 100 & 122 & & 222 \\
San Pedro Sula & & & 595 & 595 \\
Santa Bárbara & 107 & & 73 & 12 \\
Santa Rosa de Copán & 218 & & 1115 & 180 \\
Tegucigalpa & 897 & 198 & 1977 \\
\hline Total & & & 3072 \\
\hline Nota. Elaboración propia, a partir de HISTORIAL y FICHA. EFGP=Estudios de Fundamento General y \\
Pedagógicos.
\end{tabular}

Al considerar las variables demográficas de los estudiantes en la muestra (ver Tabla 2), de los 3,072 estudiantes que en 2017 ingresaron a la universidad, el 71.1\% son mujeres. La edad promedio de las mujeres es de 22.1 años, mientras que la de los hombres es de 23.4 años. Respecto a estas variables se puede decir que las mayores diferencias entre mujeres y hombres están en el porcentaje de los que se identifican como mestizos (4.5 puntos porcentuales a favor de las mujeres).

Con relación a las variables que determinan las condiciones económicas se puede afirmar que las mujeres proceden de hogares con mayores carencias, ya que un porcentaje mayor afirma tener dependientes e ingresos familiares menores a los L. 6,000. Por otra parte, el porcentaje de mujeres que dependen de sus padres supera en 6.1 puntos porcentuales al de los hombres.

Con relación a los antecedentes académicos, casi todos los porcentajes son mayores para las mujeres, salvo el de aquellas que estudiaron o actualmente estudian en otra universidad (10 puntos porcentuales menos que los hombres). Resalta el hecho que el porcentaje de mujeres que egresó de una escuela normal es mayor en 6.5 puntos porcentuales que el de hombres y el hecho que el porcentaje de mujeres recién graduadas es mayor que el de hombres en 3.5 puntos porcentuales.

Respecto a las variables de selección de acuerdo al test de aptitud académica, todos los promedios en las escalas son mayores para los hombres. Además, el porcentaje de hombres que fue seleccionado por la primera opción de carrera es mayor que el de mujeres en 2.4 puntos 
porcentuales. Finalmente, el porcentaje de hombres que eligieron estudiar en modalidad presencial es mayor en 1.3 puntos porcentuales respecto a las mujeres.

\section{Tabla 2}

Porcentaje de estudiantes según variables del perfil de ingreso y puntaje promedio de edad y en las escalas para ESPA, MATE y RAAB, según sexo.

\begin{tabular}{|c|c|c|}
\hline & Femenino & Masculino \\
\hline Total de estudiantes & 2183 & 889 \\
\hline \multicolumn{3}{|l|}{ Condición demográfica } \\
\hline Soltero & 85.6 & 84.5 \\
\hline Se identifica como mestizo & 47.2 & 42.7 \\
\hline Pertenece a un grupo originario, garífuna o negro inglés & 15.5 & 18.2 \\
\hline No sabe su grupo poblacional pertenece & 37.3 & 39.1 \\
\hline Edad promedio & 22.1 & 23.4 \\
\hline \multicolumn{3}{|l|}{ Condición económica } \\
\hline Trabaja & 29.4 & 43.3 \\
\hline Tiene dependientes & 42.4 & 41.2 \\
\hline Ingreso familiar $<$ L. 6,000 & 49.5 & 43.8 \\
\hline Depende de sus padres & 68.3 & 62.2 \\
\hline \multicolumn{3}{|l|}{ Antecedentes académicos } \\
\hline Primaria o menos (Madre) & 47.7 & 45.9 \\
\hline Estudió en colegio presencial & 90.0 & 88.8 \\
\hline Estudió en colegio público & 73.8 & 73.0 \\
\hline Estudió en Escuela Normal & 27.6 & 21.1 \\
\hline Es recién graduado & 32.0 & 28.5 \\
\hline Estudió en otra universidad & 18.5 & 26.1 \\
\hline Estudia en otra universidad & 5.0 & 7.4 \\
\hline \multicolumn{3}{|l|}{ Admisión } \\
\hline ESPA promedio* & 498.5 & 521.4 \\
\hline MATE promedio* & 489.5 & 535.3 \\
\hline RAAB promedio* & 500.7 & 524.3 \\
\hline Fue seleccionado por primera opción & 89.1 & 91.5 \\
\hline Estudiará en modalidad presencial & 70.5 & 71.8 \\
\hline
\end{tabular}

Nota. Elaboración propia, a partir de HISTORIAL y FICHA. * Puntaje promedio en la escala según sexo.

Instituto de Investigación y Evaluación Educativas y Sociales | Universidad Pedagógica Nacional Francisco Morazán 75 


\section{Relación entre el Test de Admisión y el Rendimiento en FFE0301 y FFM1301}

Para estudiar la relación de las puntuaciones de las escalas ESPA, MATE y RAAB con las calificaciones obtenidas en FFE0301 y FFM1301, se clasificó a los estudiantes por clúster para cada escala mediante el método de k medias; el número de estudiantes y los centros por clúster, se muestran en la Tabla 3 . Como se observa, los clústeres 1 agrupan a más de un tercio de la muestra; bastante si se considera que estos corresponden a los puntajes más bajos en las escalas.

\section{Tabla 3}

Número de estudiantes y centro para cada clúster según escala.

\begin{tabular}{ccccccc}
\hline & \multicolumn{7}{c}{ Clúster } \\
\cline { 2 - 7 } Escala & \multicolumn{2}{c}{$\mathbf{1}$} & \multicolumn{2}{c}{$\mathbf{2}$} & \multicolumn{3}{c}{$\mathbf{3}$} \\
\cline { 2 - 7 } & $\mathbf{n}$ & Centro & $\mathbf{n}$ & Centro & $\mathbf{n}$ & Centro \\
\hline ESPA & 1018 & 402.1 & 1440 & 515.6 & 614 & 651.2 \\
MATE & 1163 & 415.3 & 1532 & 522.7 & 377 & 690.9 \\
RAAB & 1399 & 421.2 & 994 & 521.9 & 679 & 664.4 \\
\hline
\end{tabular}

Nota. Elaboración propia.

El total de estudiantes de la muestra que tomó FFE0301 al menos una vez en los tres años académicos que incluye este estudio es de 2,680, mientras que los que tomaron FFM1301 fueron 2,332. En la Tabla 4 se muestran las estadísticas descriptivas para las calificaciones en FFE0301 y FFM1301 para cada clúster según escala. Para determinar si las diferencias observadas en las medias por clúster son estadísticamente significativas, se realizó una prueba ANOVA con contraste post-hoc. El estadístico de Levene indica que para FFE0301 las muestras obtenidas por los clústeres para ESPA y RAAB proceden de poblaciones con varianzas iguales, mientras que el resto proceden de poblaciones con varianzas diferentes; por tal razón, para las dos primeras el contraste se realizó mediante Tukey-b, mientras que para el resto se realizó un contraste mediante la prueba T2 de Tamhane. Los resultados muestran que todas las diferencias de medias son estadísticamente significativas al 95\% de confianza $(\mathrm{p}=0.000)$. Se observa que las diferencias de medias entre clúster son mayores en FFM1301 que en FFE0301. 


\section{Tabla 4}

Estadísticos descriptivos para las calificaciones en FFE0301 y FFM1301 por clúster según escala.

\begin{tabular}{cccccc}
\hline \multirow{2}{*}{ Escala } & \multirow{2}{*}{ Clúster } & \multicolumn{2}{c}{ FFM0301 } & \multicolumn{2}{c}{ FFM1301 } \\
\cline { 2 - 5 } & & Media & DT & Media & DT \\
\hline \multirow{3}{*}{ ESPA } & 1 & 73.3 & 14.46 & 54.8 & 18.15 \\
& 2 & 77.4 & 13.53 & 64.4 & 17.30 \\
& 3 & 80.5 & 13.01 & 72.4 & 18.21 \\
\multirow{2}{*}{ MATE } & 1 & 73.4 & 14.97 & 56.2 & 18.18 \\
& 2 & 78.0 & 12.74 & 64.6 & 17.76 \\
& 3 & 81.7 & 13.81 & 78.3 & 15.31 \\
\multirow{2}{*}{ RAAB } & 1 & 74.2 & 14.36 & 57.2 & 18.48 \\
& 2 & 77.7 & 13.00 & 64.5 & 17.47 \\
& 3 & 80.1 & 13.81 & 72.4 & 17.41 \\
\hline
\end{tabular}

Nota. Elaboración propia. DT = Desviación típica

\section{Trayectoria Académica Real}

Entre todas las posibilidades para diagramar la trayectoria real de los estudiantes en la muestra, en la Figura 1 se enfatiza en el grupo de estudiantes que desde su ingreso en el primer período de 2017 se matricularon al menos en un período académico en los años siguientes y si matricularon o no doce o más espacios pedagógicos.

Uno de los elementos que destaca es que el 4\% de los estudiantes abandonaron en el mismo período que ingresaron; quedando 2,950 estudiantes activos en 2017. De estos, el 45.4\% matriculó 12 espacios pedagógicos o más. Al comparar el desempeño de los que matricularon 12 o más y los que no, se observa que el porcentaje de estudiantes que aprobó todos los espacios pedagógicos es mayor en este primer grupo. Vale destacar que, de hecho, todos los indicadores desfavorables, como reprobación y abandono, presentan valores mayores para los estudiantes que matriculan menos de doce espacios pedagógicos. Adicionalmente, algunos de los estudiantes que en 2017 matricularon menos de doce espacios pedagógicos, en 2018 matricularon doce o más; sin embargo, en comparación con el grupo equivalente para 2018, también presentan mayores valores en los indicadores desfavorables.

De lo anterior se concluye que los estudiantes que desde el primer año matriculan pocos espacios pedagógicos, tienden a continuar con indicadores desfavorables al éxito académico. No obstante, se observa que los que logran mantenerse activos y matriculando doce o más espacios pedagógicos, al pasar de un año a otro presentan una tendencia creciente en el porcentaje de estudiantes que aprueban todos los espacios pedagógicos. 


\section{Figura 1}

Trayectoria real de los estudiantes de la muestra, 2017-2019.

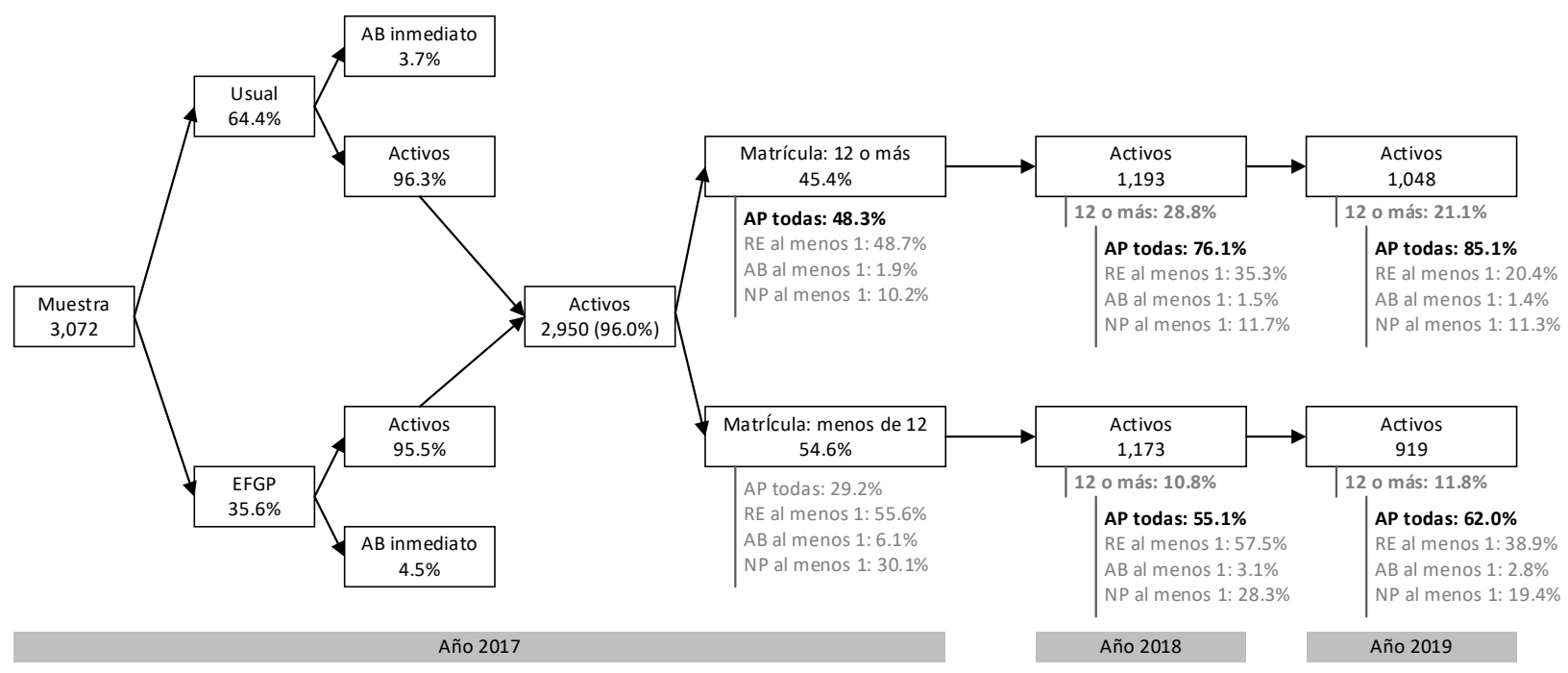

Nota. Usual=No ingresaron a EFGP; EFGP=Estudios de formación general y pedagógica; Activos=Estudiantes

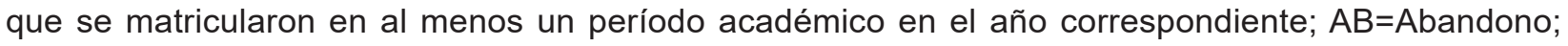
$\mathrm{AP}=$ Aprobado; RE=Reprobado; NP=No se presentó.

\section{Matrícula General por Año}

De los estudiantes en la muestra, únicamente el 29.5\% tuvieron una matrícula regular; es decir, se matricularon en los nueve períodos académicos que corresponden a los tres años incluidos en el análisis. Por otra parte, de los estudiantes que no tuvieron matrícula regular, algunos se matricularon en al menos en un período académico, pero no necesariamente en todos los años: el 96.5\% para 2017, el 74.7\% para 2018 y el 65\% para 2019. De estos estudiantes, el 59.2\% reportan matrícula en al menos un período en cada año. De lo anterior se concluye que el comportamiento de la matrícula de los estudiantes de la cohorte 2017 es irregular, lo cual podría implicar dificultades importantes al tratar de identificar variables predictoras del éxito académico.

\section{Abandono}

De los 3,072 estudiantes en la muestra, 122 (4\%) abandonaron la universidad de forma inmediata; es decir, se retiraron de la universidad en el mismo período que ingresaron. Se analizó la distribución de estudiantes al cruzar las variables clúster al que pertenece y abandono inmediato, solicitando los estadísticos para verificar la hipótesis de independencia y no se encontró relación estadísticamente significativa $\left(\chi^{2} 2=0.484, \mathrm{gl}=2, \mathrm{p}=0.785 ;\right.$ lambda $\left.=0.000\right)$. 
Por lo que se concluye que las puntuaciones en el test de aptitud académica no explican este tipo de abandono.

También, de la muestra, el 33.8\% abandonó los estudios después del último período de 2019. Se construyó un modelo de regresión logística para la variable abandono con el método Hacia atrás: Wald, con 0.05 como probabilidades para entrar o ser eliminado en el método por pasos. De acuerdo con las pruebas estadísticas, el modelo se ajusta a los datos (Hosmer y Lemeshow: $\chi 2=4.460, \mathrm{gl}=7, \mathrm{p}=0.726$, especificidad de $91.1 \%$ y sensibilidad de $85.7 \%$; R2 de Cox y Snell, 0.491; R2 de Nagelkerke, 0.680; ómnibus: $\chi 2=2074.521$, gl=5, p=0.000) (Aguayo, s.f.; Hosmer \& Lemeshow, 1989). Las variables éxito en 2018, haberse matriculado en 2018 y en 2019 y haber realizado algún tipo de cambio respecto a la preselección, reducen la probabilidad de abandono, mientras que tener más de 38 años aumenta la probabilidad de abandono (Tabla 5).

\section{Tabla 5}

Modelo de regresión logística para la variable abandono a partir de 2020.

\begin{tabular}{lcccccccc}
\hline \multicolumn{1}{c}{ Variables en el modelo } & B & EE & Wald & gl & Sig. & Exp(B) & \begin{tabular}{c} 
95\% C.I. para EXP(B) \\
\cline { 7 - 9 }
\end{tabular} \\
\hline Más de 38 años & 0.440 & 0.133 & 11.0 & 1 & 0.001 & 1.55 & 1.20 & 2.01 \\
Éxito en 2018 & -0.741 & 0.278 & 7.1 & 1 & 0.008 & 0.48 & 0.28 & 0.82 \\
Se matriculó en 2018 & -1.600 & 0.143 & 125.1 & 1 & 0.000 & 0.20 & 0.15 & 0.27 \\
Se matriculó en 2019 & -3.403 & 0.127 & 716.6 & 1 & 0.000 & 0.03 & 0.03 & 0.04 \\
Realizó algún tipo de cambio & -0.532 & 0.151 & 12.4 & 1 & 0.000 & 0.59 & 0.44 & 0.79 \\
Constante & 2.386 & 0.135 & 310.3 & 1 & 0.000 & 10.87 & & \\
\hline
\end{tabular}

Nota. Elaboración propia.

\section{Movilidad}

Algunos estudiantes de la muestra realizaron cambios respecto a la preselección: el 7.9\% cambió de centro universitario, el 10.2\% de modalidad y el $11.9 \%$ de carrera. En total el 76.6\% de los estudiantes permanecieron sin cambios a lo largo de los tres años. Respecto a los dos primeros tipos de cambios, ninguna variable del perfil de ingreso ni de la trayectoria académica es capaz de predecir la decisión de realizar algún tipo de cambio. 
Se realizó una prueba de independencia entre las variables opción de carrera con la que fue preseleccionado y realizar cambio de carrera. Los resultados muestran que si existe asociación entre estas variables $(\chi 2=125.646 ; \mathrm{gl}=1 ; \mathrm{p}=0.000)$ pero no en el sentido esperado ya que quienes fueron preseleccionado por la primera opción tienen mayor probabilidad de realizar un cambio. Vale destacar que, del total de estudiantes de la muestra, solo 313 fueron preseleccionados por la segunda opción y que todos corresponden a los centros de Tegucigalpa y San Pedro Sula (los dos con mayor matrícula a nivel nacional); de estos 98 realizaron cambio de carrera.

\section{Indicadores de Rendimiento de los Estudiantes con Matrícula Regular e Irregular}

Para efectos de este estudio se entiende como estudiante regular, aquellos que se matricularon los nueve periodos académicos correspondientes a los tres años analizados en este estudio.

De acuerdo con la Tabla 6, al comparar los estudiantes regulares con los irregulares en los tres años, como era de esperar, las medias de cursos aprobados son mayores para los regulares y las diferencias son estadísticamente significativas para todos los casos $(\mathrm{p}=0.000)$; a pesar de que estas son decrecientes por año. En cuanto a la media de cursos reprobados, únicamente en 2017 es mayor para los irregulares y la diferencia es estadísticamente significativa $(\mathrm{p}=0.000)$. Por otra parte, las diferencias de medias para abandono son cero, salvo para 2017 la cual es muy baja pero estadísticamente significativa. Con relación a no presentarse al curso, aunque las diferencias de medias son bastante pequeñas, todas son estadísticamente significativas ( $\mathrm{p}=0.000$ para 2017 y 2019; $\mathrm{p}=0.001$ para 2018).

Se destaca el hecho que es que las medias de cursos aprobados forman una sucesión decreciente a lo largo de los años, independientemente del tipo de estudiante. Esto significa que la eficiencia terminal se ve afectada incluso en el grupo de estudiantes regulares.

\section{Tabla 6}

Estadísticas descriptivas de los estatus de los espacios pedagógicos matriculados por estudiantes irregulares y regulares según año académico.

\begin{tabular}{lcccc}
\hline & \multicolumn{2}{c}{ Irregulares } & \multicolumn{2}{c}{ Regulares } \\
\cline { 2 - 5 } & Media & DT & Media & DT \\
\hline Aprobados & & & & \\
Reprobados & 7.2 & 4.45 & 11.3 & 2.65 \\
Abandonados & 1.1 & 1.34 & 0.8 & 1.13 \\
No se presentó & 0.1 & 0.26 & 0.0 & 0.11 \\
\hline
\end{tabular}


Continúa Tabla 6

\begin{tabular}{lcccc}
\hline & \multicolumn{2}{c}{ Irregulares } & \multicolumn{2}{c}{ Regulares } \\
\cline { 2 - 5 } & Media & DT & Media & DT \\
\hline Aprobados & & & & \\
Reprobados & 5.8 & 3.65 & 9.4 & 1.83 \\
Abandonados & 1.8 & 1.06 & 1.8 & 0.21 \\
No se presentó & 1.0 & 0.21 & 1.0 & 0.77 \\
2019 & 1.8 & 1.10 & 1.4 & \\
Aprobados & & & & 2.81 \\
Reprobados & 5.4 & 3.54 & 8.9 & 1.08 \\
Abandonados & 1.7 & 0.93 & 1.7 & 0.00 \\
No se presentó & 1.1 & 0.26 & 1.0 & 0.76 \\
\hline
\end{tabular}

Nota. Elaboración propia. DT = Desviación típica

\section{El Éxito Académico}

Para el estudio del éxito académico se construyeron modelos de regresión logística para cada año analizado, incluyendo como predictores las variables del perfil de ingreso y algunas de la trayectoria académica. Se examinó la matriz de correlaciones de las variables predictoras a fin de identificar posibles interacciones; salvo para algunos grupos de variables dicotomizadas, todas las correlaciones resultaron inferiores a 0.3 . Por otra parte, no se identificó problemas de colinealidad, ya que tanto el factor de inflación de la varianza, como los índices de condición y la descomposición de la varianza así lo confirman (Akinwande, et al., 2015; López, 1998).

\section{Éxito Académico en 2017}

Para la construcción del modelo de regresión logística para predecir el éxito en 2017 se incluyó a 1,006 estudiantes que iniciaron en la Facultad de Ciencia y Tecnología (FACYT), de los cuales el 37\% aprobó 12 o más cursos en ese año. Por otra parte, para el modelo para la Facultad de Humanidades (FAHU) se incluyó a 1,672 estudiantes, de los cuales el 34\% aprobó 12 o más cursos en ese año.

Según se muestra en la Tabla 7, ser menor de 38 años, haberse graduado de media en 2016, obtener calificaciones de $70 \%$ o más en 2017 , haber sido preseleccionado con la primera opción de carrera, depender de un familiar que no sean sus padres, ser estudiante regular y obtener puntajes cercanos a la media en la escala de matemáticas del test de aptitud académica, favorecen el éxito en el primer año de estudios para los estudiantes de la FACYT. En cambio, para la FAHU, el éxito académico en el primer año de estudios se ve favorecido por tener menos de 38 años, 
pertenecer a un grupo originario (o garífuna o negro inglés), tener título de maestro de educación primaria, ser preseleccionado con la primera opción de carrera y ser un estudiante regular.

\section{Tabla 7}

Modelos de regresión logística para el éxito académico en 2017 por facultad.

\begin{tabular}{|c|c|c|c|c|c|c|c|c|}
\hline & \multirow[t]{2}{*}{ B } & \multirow{2}{*}{$\begin{array}{c}\text { Error } \\
\text { estándar }\end{array}$} & \multirow[t]{2}{*}{ Wald } & \multirow[t]{2}{*}{ gl } & \multirow[t]{2}{*}{ Sig. } & \multirow[t]{2}{*}{$\operatorname{Exp}(B)$} & \multicolumn{2}{|c|}{$\begin{array}{c}\text { 95\% C.I. para } \\
\text { EXP(B) }\end{array}$} \\
\hline & & & & & & & Inferior & Superior \\
\hline \multicolumn{9}{|l|}{ FACYT } \\
\hline Edad entre 31 a 38 años & 1.202 & 0.419 & 8.219 & 1 & 0.004 & 3.326 & 1.463 & 7.564 \\
\hline Edad mayor que 38 años & -0.521 & 0.230 & 5.139 & 1 & 0.023 & 0.594 & 0.379 & 0.932 \\
\hline Se identifica como mestizo & -0.480 & 0.254 & 3.580 & 1 & 0.058 & 0.619 & 0.377 & 1.017 \\
\hline No sabe a cuál grupo poblacional pertenece & -0.521 & 0.264 & 3.898 & 1 & 0.048 & 0.594 & 0.354 & 0.996 \\
\hline Depende de algún familiar & 1.043 & 0.597 & 3.049 & 1 & 0.081 & 2.836 & 0.880 & 9.140 \\
\hline Egresó de una escuela normal & 0.544 & 0.217 & 6.292 & 1 & 0.012 & 1.723 & 1.126 & 2.635 \\
\hline Recién graduado & 0.734 & 0.217 & 11.504 & 1 & 0.001 & 2.084 & 1.363 & 3.186 \\
\hline Estudió en alguna universidad & -0.577 & 0.226 & 6.521 & 1 & 0.011 & 0.562 & 0.361 & 0.874 \\
\hline Índice académico en 2017 igual o mayor a 70 & 5.121 & 0.595 & 74.038 & 1 & 0.000 & 167.482 & 52.166 & 537.712 \\
\hline Fue preseleccionado por la primera opción & 1.776 & 0.346 & 26.406 & 1 & 0.000 & 5.905 & 2.999 & 11.623 \\
\hline Está en el clúster 2 de MATE & 0.560 & 0.178 & 9.952 & 1 & 0.002 & 1.751 & 1.236 & 2.481 \\
\hline Se matriculó en todos los períodos académicos & 0.953 & 0.179 & 28.357 & 1 & 0.000 & 2.594 & 1.827 & 3.685 \\
\hline Constante & -6.934 & 0.727 & 90.945 & 1 & 0.000 & 0.001 & & \\
\hline \multicolumn{9}{|l|}{ FAHU } \\
\hline Edad mayor que 38 años & -0.500 & 0.148 & 11.395 & 1 & 0.001 & 0.607 & 0.454 & 0.811 \\
\hline $\begin{array}{l}\text { Pertenece a un grupo originario, garífuna o negro } \\
\text { inglés }\end{array}$ & 0.563 & 0.193 & 8.546 & 1 & 0.003 & 1.757 & 1.204 & 2.563 \\
\hline Es mestizo & 0.441 & 0.135 & 10.633 & 1 & 0.001 & 1.554 & 1.192 & 2.025 \\
\hline Egresó de una escuela normal & 0.888 & 0.137 & 42.107 & 1 & 0.000 & 2.430 & 1.858 & 3.177 \\
\hline Estudió en alguna universidad & -0.535 & 0.166 & 10.325 & 1 & 0.001 & 0.586 & 0.423 & 0.812 \\
\hline Fue preseleccionado por la primera opción & 0.803 & 0.221 & 13.167 & 1 & 0.000 & 2.232 & 1.446 & 3.443 \\
\hline Índice académico en 2017 igual o mayor a 70 & 3.781 & 0.365 & 107.281 & 1 & 0.000 & 43.859 & 21.445 & 89.698 \\
\hline Se matriculó en todos los períodos académicos & 0.716 & 0.126 & 32.076 & 1 & 0.000 & 2.047 & 1.597 & 2.622 \\
\hline Constante & -5.235 & 0.426 & 151.089 & 1 & 0.000 & 0.005 & & \\
\hline
\end{tabular}

Nota. Elaboración propia.

En la Tabla 8 se muestran los estadísticos de calidad de los modelos de la Tabla 7. Ambos modelos son buenos para clasificar correctamente los casos de no éxito (sensibilidad cercana a $80 \%$ ), pero menos efectivos para clasificar correctamente los casos de éxito, sobre todo el modelo para la FAHU (especificidad de 60.7\%). Por otra parte, en el caso del modelo para la FAHU, vale 
destacar que haber estudiado en alguna universidad en el pasado mejora la sensibilidad (en 1.6 puntos porcentuales) pero reduce el porcentaje de varianza (en 0.001) y la especificidad (en 0.9 puntos porcentuales) en comparación con estar estudiando en una universidad actualmente.

\section{Tabla 8}

Estadísticos acerca de la calidad de los modelos de regresión logística para el éxito en 2017 por facultad.

\begin{tabular}{ccc}
\hline Estadístico & FACYT & FAHU \\
\hline \multirow{2}{*}{ Hosmer y Lemeshow } & $\chi^{2}=5.265$ & $\chi^{2}=8.114$ \\
Sensibilidad & $\mathrm{gl}=8$ & $\mathrm{gl}=8$ \\
Especificidad & $\mathrm{p}=0.729$ & $\mathrm{p}=0.422$ \\
\cline { 2 - 3 } R2 de Cox y Snell & $80.5 \%$ & $81.5 \%$ \\
R2 de Nagelkerke & $79.2 \%$ & $60.7 \%$ \\
& 0.412 & 0.300 \\
Ómnibus & 0.562 & 0.415 \\
\hline & $\chi^{2}=534.137$ & $\chi^{2}=596.488$ \\
& $\mathrm{gl}=1$ & $\mathrm{gl}=8$ \\
& $\mathrm{p}=0.000$ & $\mathrm{p}=0.000$ \\
\hline
\end{tabular}

Nota. Elaboración propia.

\section{Éxito Académico en 2018}

Para la construcción del modelo de regresión logística para predecir el éxito en 2018 de los estudiantes de la FACYT se incluyó a 1,020 estudiantes; de los cuales el 9\% aprobó 12 o más cursos en ese año. Respecto al modelo para la FAHU, se incluyó a 1,705 estudiantes, de los cuales el 14\% aprobó 12 o más cursos en ese año.

Tal como se observa en la Tabla 9, el éxito académico para los estudiantes de la FACYT, se ve favorecido por haber estado matriculado en otra universidad al momento de ser preseleccionado, obtener calificaciones de 70\% o más en ese año, ser estudiante regular y haber sido exitoso en 2017. En cambio, el éxito académico para el segundo año académico se ve afectado negativamente por tener una edad de 19 a 30 años, haber estado trabajando al momento de la preselección, ser maestro de educación primaria y realizar un cambio de centro regional.

El éxito académico para estudiantes de la FAHU se ve favorecido por ser de sexo masculino, haber estudiado en la modalidad presencial en educación media, obtener calificaciones de 70\% o más 
en ese año, haber obtenido un puntaje en la escala de matemáticas (del test de aptitud académica) entre 400 y 500, ser estudiante regular y haber sido exitoso en 2017. Por otra parte, el hecho de cambiar de centro regional o de carrera afecta negativamente el éxito académico.

\section{Tabla 9}

Modelos de regresión logística para el éxito académico en 2018 por facultad.

\begin{tabular}{|c|c|c|c|c|c|c|c|c|}
\hline & \multirow{2}{*}{ B } & \multirow{2}{*}{$\begin{array}{c}\text { Error } \\
\text { estándar }\end{array}$} & \multirow{2}{*}{ Wald } & \multirow{2}{*}{ gl } & \multirow{2}{*}{ Sig. } & \multirow{2}{*}{$\operatorname{Exp}(B)$} & \multicolumn{2}{|c|}{$\begin{array}{c}\text { 95\% C.I. para } \\
\text { EXP(B) } \\
\end{array}$} \\
\hline & & & & & & & Inferior & Superior \\
\hline \multicolumn{9}{|l|}{ FACYT } \\
\hline Edad de 19 a 24 años & -0.607 & 0.286 & 4.497 & 1 & 0.034 & 0.545 & 0.311 & 0.955 \\
\hline Edad de 25 a 30 años & -1.452 & 0.669 & 4.708 & 1 & 0.030 & 0.234 & 0.063 & 0.869 \\
\hline Trabaja & -1.259 & 0.365 & 11.906 & 1 & 0.001 & 0.284 & 0.139 & 0.580 \\
\hline Egresó de una escuela normal & -0.984 & 0.361 & 7.442 & 1 & 0.006 & 0.374 & 0.184 & 0.758 \\
\hline Estudia en alguna universidad & 1.364 & 0.495 & 7.598 & 1 & 0.006 & 3.912 & 1.483 & 10.318 \\
\hline Cambió de centro de estudio & -1.907 & 0.648 & 8.675 & 1 & 0.003 & 0.148 & 0.042 & 0.528 \\
\hline Índice académico en 2017 igual o mayor a 70 & 2.794 & 0.319 & 76.518 & 1 & 0.000 & 16.344 & 8.740 & 30.566 \\
\hline Se matriculó en todos los períodos académicos & 1.003 & 0.294 & 11.615 & 1 & 0.001 & 2.726 & 1.531 & 4.853 \\
\hline Aprobó 12 o más espacios pedagógicos en 2017 & 0.986 & 0.293 & 11.354 & 1 & 0.001 & 2.681 & 1.511 & 4.758 \\
\hline Constante & -4.060 & 0.356 & 129.886 & 1 & 0.000 & 0.017 & & \\
\hline \multicolumn{9}{|l|}{ FAHU } \\
\hline Masculino & 0.526 & 0.180 & 8.493 & 1 & 0.004 & 1.692 & 1.188 & 2.410 \\
\hline Estudió en modalidad presencial en media & 0.853 & 0.366 & 5.441 & 1 & 0.020 & 2.347 & 1.146 & 4.808 \\
\hline Realizó cambió de centro regional & -1.343 & 0.408 & 10.815 & 1 & 0.001 & 0.261 & 0.117 & 0.581 \\
\hline Realizó cambió de carrera & -1.336 & 0.413 & 10.457 & 1 & 0.001 & 0.263 & 0.117 & 0.591 \\
\hline Índice académico en 2018 igual o mayor a 70 & 2.166 & 0.221 & 96.162 & 1 & 0.000 & 8.724 & 5.659 & 13.451 \\
\hline Su puntaje en MATE está entre 400 y 500 & 0.373 & 0.165 & 5.127 & 1 & 0.024 & 1.452 & 1.051 & 2.006 \\
\hline Se matriculó en todos los períodos académicos & 0.794 & 0.170 & 21.815 & 1 & 0.000 & 2.213 & 1.586 & 3.089 \\
\hline Aprobó 12 o más espacios pedagógicos en 2017 & 0.998 & 0.169 & 34.668 & 1 & 0.000 & 2.712 & 1.946 & 3.780 \\
\hline Constante & -5.026 & 0.428 & 138.152 & 1 & 0.000 & 0.007 & & \\
\hline
\end{tabular}

Nota. Elaboración propia.

En la Tabla 10 se muestran los estadísticos de calidad de los modelos de la Tabla 9. Ambos modelos son buenos para clasificar correctamente los casos de no éxito (sensibilidad entre 96\% y 
98\%), pero muy poco efectivos para clasificar correctamente los casos de éxito, sobre todo el modelo para la FAHU (especificidad de 23.9\%).

\section{Tabla 10}

Estadísticos acerca de la calidad de los modelos de regresión logística para el éxito en 2018 por facultad.

\begin{tabular}{ccc}
\hline Estadístico & FACYT & FAHU \\
\hline \multirow{2}{*}{ Hosmer y Lemeshow } & $\chi^{2}=14.365$ & $\chi^{2}=13.630$ \\
& $\mathrm{gl}=8$ & $\mathrm{gl}=7$ \\
Sensibilidad & $\mathrm{p}=0.073$ & $\mathrm{p}=0.058$ \\
\cline { 2 - 3 } Especificidad & $98.1 \%$ & $96.5 \%$ \\
$\mathrm{R}^{2}$ de Cox y Snell & $41.3 \%$ & $23.9 \%$ \\
$\mathrm{R}^{2}$ de Nagelkerke & 0.202 & 0.193 \\
& 0.445 & 0.351 \\
\cline { 2 - 3 } Ómnibus & $\chi^{2}=230.208$ & $\chi^{2}=336.550$ \\
& $\mathrm{gl}=9$ & $\mathrm{gl}=8$ \\
& $\mathrm{p}=0.000$ & $\mathrm{p}=0.000$ \\
\hline
\end{tabular}

Nota. Elaboración propia.

\section{Éxito Académico en 2019}

El modelo de regresión logística para predecir el éxito en 2019 incluyó a 1,020 estudiantes que iniciaron en la FACYT, de los cuales el 5\% aprobó 12 o más cursos en ese año. Para el modelo de la FAHU se incluyó a 1,705 estudiantes, de los cuales el 12\% aprobó 12 o más cursos en ese año.

Para los estudiantes de la FACYT, el éxito académico se ve favorecido por ser de un grupo originario (o garífuna o negro inglés), haber estudiado en una universidad en el pasado, obtener calificaciones de 70\% o más en ese año, ser estudiante regular y haber sido exitoso en 2018. En cambio, haber ingresado con una edad de 19 a 24 años, haber estado trabajando al momento de la preselección, ser maestro de educación primaria afecta negativamente el éxito académico. Al considerar el modelo de la FAHU, se encontró un impacto positivo al tener un ingreso familiar de más de 9,000 lempiras al momento de la preselección, haberse graduado en 2016, ser estudiante regular, obtener calificaciones de 70\% o más en 2018 y en 2019 y ser exitoso en 2017 y en 2018. Por otra parte, tener hijos al momento de ser preseleccionado y realizar cambios de centro regional y de carrera son desfavorables al éxito académico (ver Tabla 11). 


\section{Tabla 11}

Modelos de regresión logistica para el éxito académico en 2019 por facultad.

\begin{tabular}{|c|c|c|c|c|c|c|c|c|}
\hline & B & Error & Wald & gl & Sig. & $\operatorname{Exp}(B)$ & $\begin{array}{r}95 \% \mathrm{C} \\
\text { EX } \\
\end{array}$ & $\begin{array}{l}\text { I. para } \\
\mathbf{P}(\mathbf{B})\end{array}$ \\
\hline & & & & & & & Inferior & Superior \\
\hline FACYT & & & & & & & & \\
\hline Edad de 19 a 24 años & -1.031 & 0.416 & 6.154 & 1 & 0.013 & 0.357 & 0.158 & 0.805 \\
\hline Pertenece a un grupo originario, garífuna o negro inglés & 1.165 & 0.444 & 6.890 & 1 & 0.009 & 3.205 & 1.343 & 7.647 \\
\hline Trabaja & -1.985 & 0.654 & 9.211 & 1 & 0.002 & 0.137 & 0.038 & 0.495 \\
\hline Egresó de una escuela normal & -0.975 & 0.505 & 3.724 & 1 & 0.054 & 0.377 & 0.140 & 1.015 \\
\hline Estudió en alguna universidad & 0.731 & 0.441 & 2.752 & 1 & 0.097 & 2.077 & 0.876 & 4.928 \\
\hline Índice académico en 2019 igual o mayor a 70 & 1.634 & 0.397 & 16.926 & 1 & 0.000 & 5.125 & 2.353 & 11.164 \\
\hline Se matriculó en todos los períodos académicos & 2.005 & 0.479 & 17.484 & 1 & 0.000 & 7.423 & 2.901 & 18.996 \\
\hline Aprobó 12 o más espacios pedagógicos en 2018 & 1.372 & 0.385 & 12.732 & 1 & 0.000 & 3.944 & 1.856 & 8.380 \\
\hline Constante & -4.962 & 0.516 & 92.374 & 1 & 0.000 & 0.007 & & \\
\hline FAHU & & & & & & & & \\
\hline Ingreso familiar de L. 6,001 a L. 9,000 & 0.563 & 0.220 & 6.529 & 1 & 0.011 & 1.756 & 1.140 & 2.704 \\
\hline Ingreso familiar de más de L. 12,000 & 0.628 & 0.256 & 6.021 & 1 & 0.014 & 1.874 & 1.135 & 3.095 \\
\hline Tiene hijos & -0.613 & 0.295 & 4.335 & 1 & 0.037 & 0.542 & 0.304 & 0.965 \\
\hline Recién graduado & 0.764 & 0.279 & 7.524 & 1 & 0.006 & 2.147 & 1.244 & 3.706 \\
\hline De 1 a 2 años de haberse graduado & 0.690 & 0.276 & 6.267 & 1 & 0.012 & 1.994 & 1.162 & 3.423 \\
\hline De 6 a 10 años de haberse graduado & 0.812 & 0.355 & 5.228 & 1 & 0.022 & 2.252 & 1.123 & 4.516 \\
\hline Cambió de centro de estudio & -1.287 & 0.464 & 7.690 & 1 & 0.006 & 0.276 & 0.111 & 0.686 \\
\hline Cambió de carrera & -1.271 & 0.464 & 7.491 & 1 & 0.006 & 0.281 & 0.113 & 0.697 \\
\hline Se matriculó en todos los períodos académicos & 0.872 & 0.200 & 19.089 & 1 & 0.000 & 2.392 & 1.618 & 3.538 \\
\hline Índice académico en 2018 igual o mayor a 70 & 0.855 & 0.252 & 11.565 & 1 & 0.001 & 2.352 & 1.437 & 3.851 \\
\hline Índice académico en 2019 igual o mayor a 70 & 2.569 & 0.328 & 61.442 & 1 & 0.000 & 13.052 & 6.866 & 24.812 \\
\hline Aprobó 12 o más espacios pedagógicos en 2017 & 0.484 & 0.199 & 5.938 & 1 & 0.015 & 1.622 & 1.099 & 2.394 \\
\hline Aprobó 12 o más espacios pedagógicos en 2018 & 1.177 & 0.199 & 34.898 & 1 & 0.000 & 3.244 & 2.196 & 4.794 \\
\hline Constante & -5.941 & 0.418 & 201.589 & 1 & 0.000 & 0.003 & & \\
\hline
\end{tabular}

Nota. Elaboración propia.

Los modelos anteriores son excelentes para identificar casos de no éxito para ambas facultades (sensibilidad entre 96\% y 99\%), pero no tanto para clasificar casos de éxito (especificidad cercana al 40\%); lo cual se aprecia en la Tabla 12. 
Tabla 12

Estadísticos acerca de la calidad de los modelos de regresión logística para el éxito en 2019 por facultad.

\begin{tabular}{ccc}
\hline Estadístico & FACYT & FAHU \\
\hline \multirow{2}{*}{ Hosmer y Lemeshow } & $\chi^{2}=4.024$ & $\chi^{2}=6.438$ \\
& $\mathrm{gl}=8$ & $\mathrm{gl}=8$ \\
Sensibilidad & $\mathrm{p}=0.855$ & $\mathrm{p}=0.598$ \\
\cline { 2 - 3 } Especificidad & $98.9 \%$ & $96.6 \%$ \\
$\mathrm{R}^{2}$ de Cox y Snell & $39.1 \%$ & $42.2 \% ;$ \\
$\mathrm{R}^{2}$ de Nagelkerke & 0.127 & 0.253 \\
& 0.413 & 0.486 \\
\cline { 2 - 3 } Ómnibus & $\chi^{2}=138.532$ & $\chi^{2}=497.997$ \\
& $\mathrm{gl}=8$ & $\mathrm{gl}=13$ \\
& $\mathrm{p}=0.000$ & $\mathrm{p}=0.000$ \\
\hline
\end{tabular}

Nota. Elaboración propia.

\section{Conclusiones}

El estudio de la trayectoria académica resulta relevante para las universidades, pues brinda información acerca de la eficiencia terminal y del comportamiento de los indicadores que la explican. Desafortunadamente, debido a que el comportamiento de las trayectorias reales se explica de manera multifactorial y a que estas pueden diferir substancialmente de las trayectorias teóricas, su estudio representa un verdadero reto.

En este estudio se encontró que un número reducido de variables del perfil de ingreso explica la trayectoria académica, y que la influencia de las mismas difiere por año académico y por facultad a la cual están adscritos los estudiantes. Sin embargo, a medida que se avanza en los años, las variables del desempeño académico de los años anteriores son fuertes predictores del éxito en el año siguiente.

\section{EI Perfil de Ingreso}

1. Con relación al perfil de ingreso y condición de admisión, se encontró que el mayor porcentaje de estudiantes son mujeres, las cuales provienen de hogares más desfavorecidos económicamente, con mayor dependencia económica de los padres y con puntajes más bajos en las escalas del test de aptitud académica. Sin embargo, también se encontró que la variable sexo no tiene impacto importante en el éxito académico. 


\section{El Test de Admisión y el Rendimiento en FFE0301 y FFM1301}

2. El test de aptitud académica, es predictor del desempeño en FFE0301 y FFM1301; a mayor puntaje en el test de aptitud académica mayor rendimiento, especialmente en FFM1301.

\section{Las Trayectorias Académicas}

3. Las trayectorias académicas reales de los estudiantes distan enormemente de las teóricas, principalmente debido a lo irregular de la matrícula en cada período académico por año, a la movilidad, la reprobación y al abandono. Algunos de estos indicadores no pueden ser explicados por las variables del perfil de ingreso. Sin embargo, el abandono después de tres años de estudio está explicado por variables relacionadas con la trayectoria en los años anteriores. Por otra parte, los estudiantes regulares presentan mejores indicadores relacionados con la trayectoria, lo cual implica mayor tasa de aprobación de cursos y menor tasa de reprobación, abandono y no presentarse; no obstante, los indicadores favorables tienen una tendencia decreciente a medida que se avanza en los años académicos.

\section{Respecto al Éxito Académico}

4. De las variables demográficas, sexo tiene muy poco impacto para explicar el éxito académico. La edad aparece más veces en los modelos, con influencia diferenciada por rango de edad y sin una tendencia. La identificación como mestizo, no saber con cuál grupo pertenece o identificarse con algún grupo originario (o garífuna o negro inglés) tiene una influencia poco uniforme en el éxito académico; aunque se destaca que identificarse en este último grupo tiene influencia positiva para los estudiantes de la FAHU.

5. Respecto a la condición económica al momento del ingreso, se puede decir que no existe un impacto tan importante en los indicadores relacionados con la trayectoria académica. La variable "Trabaja" aparece pocas veces como predictor, pero siempre con impacto negativo. Depender económicamente solo aparece una vez y con impacto positivo si "depende de algún familiar". Los rangos de ingreso aparecen únicamente en el modelo para la FAHU de 2019, siendo los mayores que L. 9,000 con un impacto positivo.

6. Con relación a los antecedentes académicos, tampoco se observa una tendencia clara. Haber egresado de una Escuela Normal tiene un impacto negativo para los estudiantes de la FACYT a partir del segundo año. Haber estudiado en una universidad en el pasado tiene un impacto negativo en el primer año para los estudiantes de ambas facultades, pero para los siguientes años no aparece de manera regular en los modelos. Haberse graduado de educación media en 2016 aparece dos veces con impacto positivo (para el modelo de la FACYT de 2017 y el de la FAHU de 2019).

7. Los buenos resultados en un año académico favorecen el éxito académico del año siguiente. Por ejemplo, no basta con aprobar los espacios pedagógicos para aumentar la probabilidad de éxito académico; es necesario obtener una calificación promedio igual o mayor que $70 \%$. 
8. Con relación a la condición de admisión, los puntajes en el test de aptitud académica no tienen un impacto importante en el éxito académico. El haber sido preseleccionado por la primera opción tiene un impacto positivo en el éxito académico del primer año en ambas facultades.

En este estudio se analizó el perfil de ingreso y la trayectoria académico de manera general para una cohorte, sin distinguir por carrera, centro regional ni modalidad. Se recomienda realizar estudios similares atendiendo estas variables, ya que podría ser de mayor utilidad para la toma de decisiones. Por otra parte, considerar la trayectoria desde la perspectiva cuantitativa implica un análisis global que no expresa en su totalidad las condiciones de la compleja situación de los estudiantes. En ese sentido, parece claro que para indagar con mayor profundidad en el tema de la trayectoria estudiantil se requiere complementar por medio de estudios con un enfoque cualitativo. Finalmente, de acuerdo con la literatura consultada, existen otros modelos estadísticos o matemáticos para el estudio de la eficiencia terminal y de las trayectorias académicas, así como otros enfoques para abordar estos estudios. Por ello, se recomienda fomentar estudios desde esta diversidad metodológica, a fin de lograr una mejor comprensión del fenómeno.

\section{Referencias Bibliográficas}

Abad, F., Olea, J., Ponsoda, V. y García, C. (2011). Medición en ciencias sociales y de la salud. Editorial Síntesis. Madrid.

Aguayo, M. (s.f.). Cómo hacer una Regresión Logística con SPSSC “paso a paso”. (I). [Archivo PDF]. http://metodos-avanzados.sociales.uba.ar/wp-content/uploads/sites/216/2014/03/Regres_ log_AGUAYO-otros.pdf

Akinwande, M.O., Dikko, H.G. \& Samson, A. (2015) Variance Inflation Factor: As a Condition for the Inclusion of Suppressor Variable(s) in Regression Analysis. Open Journal of Statistics, 5(7), 754-767. http://dx.doi.org/10.4236/ojs.2015.57075

Arita, M. (2010). Factores que inciden en los niveles de repitencia de las asignaturas de Sociología General e Historia de Honduras en la UNAH-VS. Universidad Nacional Autónoma de HondurasVS. https://tzibalnaah.unah.edu.hn/xmlui/bitstream/handle/123456789/146/T-MSc00043.pdf? sequence $=2 \&$ is Allowed $=\mathrm{y}$

Báez, M. (2013). Factores que determinan el desempeño académico en Zamorano. Universidad Zamorano. https://bdigital.zamorano.edu/bitstream/11036/1836/1/AGN-2013-T005.pdf 
Berríos, M., Medina, A., Castillo, M., González, C. y Ramos, A. (2016). Eficiencia terminal: problemática y propuesta de mejora para la carrera de turismo y hostelería desde la perspectiva estudiantil. Universidad Pedagógica Nacional Francisco Morazán. https://postgrado.upnfm.edu.hn/files/ VRIP/Eje\%20investigacion/Investigaciones/Eficiencia\%20Terminal_problematica\%20y\% 20propuesta $\% 20$ de $\% 20$ mejroa $\% 20$ para $\% 201 \mathrm{a} \% 20$ carrera $\% 20 \mathrm{de} \% 20$ Hosteler $\% \mathrm{C} 3 \%$ ADa $\%$ 20y\%20Turismo.pdf

Buela-Casal, G., Vadillo, Ó., Pagani, R., Bermúdez, M., Sierra, J., Zych, I. y Castro, Á. (2009). Comparación de los indicadores de la calidad de las universidades. RUSC. Universities and Knowledge Society Journal, 6(2), 9-21. https://www.redalyc.org/articulo.oa?id=78012947008

Castillo, J. y Martínez, J. (2016). Percepción de los estudiantes de la carrera de educación comercial respecto a las causas internas y externas que inciden o no en la eficiencia terminal de la generación 2012-2015 de la Universidad Pedagógica Nacional Francisco Morazán UPNFM sede Tegucigalpa, Honduras. [Archivo PDF] https://postgrado.upnfm.edu.hn/files/VRIP/Eje\%20 investigacion/Investigaciones/Percepci $\% \mathrm{C3} \% \mathrm{~B} 3 \mathrm{n} \% 20 \mathrm{de} \% 201 \mathrm{los} \% 20$ estudiantes $\% 20 \mathrm{de} \% 201 \mathrm{a} \%$ 20Carrera\%20de\%20Educaci\%C3\%B3n\%20Comercial\%20respecto\%20a\%201a\%20eficiencia $\%$ 20terminal.pdf

Chain Revuelta, R., Cruz Ramirez, N., Martinez Morales, M. y Jacome Ávila, N. (2003). Examen de selección y probabilidad de éxito escolar en estudios superiores. Estudio en una universidad pública estatal mexicana. Revista Electrónica de Investigación Educativa, 5(1). http://redie. uabc.mx/vol5nol/contenido-chain.html

Coordinación de Examen de Admisión (2019). El Proceso de Admisión en la UPNFM. Boletín No. 1.

Corominas, E. (2001). La transición de los estudios universitarios. Abandono o cambio en el primer año de Universidad. Revista de Investigación Educativa, 19(1). https://revistas.um.es/rie/article/ view/96361

Fischer, R. y Repetto, A. (2002). Método de selección y resultados académicos: Escuela de Ingeniería de la Universidad de Chile. Estudios Públicos, (92), 229-250. https://www.cepchile.cl/cep/site/ docs/20160304/20160304093149/rev92_fischer.pdf

García Robelo, O. y Barrón Tirado, C. (2011). Un estudio sobre la trayectoria escolar de los estudiantes de doctorado en Pedagogía. Perfiles educativos, 33(131), 94-113. http://www.scielo.org.mx/ scielo.php?script=sci_arttext\&pid=S0185-26982011000100007\&lng=es\&tlng=es.

Guevara, H. y Belelli, S. (2013). Las trayectorias académicas: dimensiones personales de una trayectoria estudiantil. Testimonio de un actor. RevIISE - Revista de Ciencias Sociales y Humanas, 4(4), 45-56. http://www.ojs.unsj.edu.ar/index.php/reviise/article/view/40 
Hosmer Jr, D. \& Lemeshow, S. (1989). Applied logistic regression. John Wiley \& Sons.

Lago, D., Gamoba, A. y Monter, A. (2014). Calidad de la educación superior: un análisis de sus principales determinantes. Saber, ciencia y libertad, 9(1), 157-170. https://dialnet.unirioja.es/ servlet/articulo?codigo $=5104971$

López Suárez, A., Albíter Rodríguez, Á. y Ramírez Revueltas, L. (2008). Eficiencia terminal en la educación superior, la necesidad de un nuevo paradigma. Revista de la educación superior, 37(146), 135-151. http://www.scielo.org.mx/scielo.php?script=sci_arttext\&pid=S0185-2760200 8000200009

López, E. (1998). Tratamiento de la colinealidad en regresión múltiple. Psicothema, 10(2), 491-507. http://www.psicothema.com/psicothema.asp?id=181

Ministerio de Educación Nacional (2008). Análisis de determinantes de la deserción en la educación superior colombiana con base en el SPADIES. Primera parte: Factores socioeconómicos. Factores académicos e institucionales. https:/www.mineducacion.gov.co/sistemasdeinformacion/1735/ articles-254702_determinantes_desercion.pdf

Olarte Moyano, J. (2020). Factores que predicen la permanencia estudiantil: análisis en un escenario de educación técnica. Cultura Educación y Sociedad, 11(1), 25-38. https://doi.org/10.17981/ cultedusoc.11.1.2020.02

Pérez Alcántara, B. (2017). Análisis de trayectorias escolares de estudiantes de la licenciatura en geografía de la UAEMEX. RIDE. Revista Iberoamericana para la Investigación y el Desarrollo Educativo, 7(14), 345-371. https://doi.org/10.23913/ride.v7i14.291

Pérez Granados, L. (2014). La selección de candidatos a la formación docente en Finlandia. La relevancia de las disposiciones personales hacia la actividad docente. Revista electrónica de investigación y docencia, (12). https://revistaselectronicas.ujaen.es/index.php/reid/article/view/1306

Pérez, S., Fiallos, Z., Rodríguez, D., Suazo, A., Flores, M., Ferrera, M. y Chinchilla, B. (2011). La eficiencia terminal en las carreras de pregrado de la modalidad presencial Sede Central de la UPNFM en las cohortes 2006 y 2007. Revista Académica de Investigación y Postgrado-Edición Especial, 151-159. https://postgrado.upnfm.edu.hn/files/VRIP/Revistas/Investigaci\%C3\%B3n \%20y\%20Postgrado\%202011.pdf

Rodríguez Garcés, C y Padilla Fuentes, G. (2016). Trayectoria Escolar y Ranking: valoraciones y estrategias institucionales en el nuevo escenario de selección universitaria. Revista Estudios Pedagógicos, 62(3), 316-326. http://dx.doi.org/10.4067/S0718-07052016000400017. 
Solórzano, M. (2005). Análisis del proceso de selección de estudiantes a la carrera de Ingeniería Forestal de la Escuela Nacional de Ciencias Forestales (ESNACIFOR) [Tesis de maestría, Universidad Pedagógica Nacional Francisco Morazán]. Repositorio Institucional de la Universidad Pedagógica Nacional Francisco Morazán. http://repositorio.upnfm.edu.hn:8081/xmlui/handle/12345678/134

Soria-Barreto, K. y Zúñiga-Jara, S. (2014). Aspectos determinantes del éxito académico de estudiantes universitarios. Formación universitaria, 7(5), 41-50. https://dx.doi.org/10.4067/S0718-5006 2014000500006

Unidad de Medición de la Calidad de la Educación (2017). Seguimiento a indicadores educativos 2016: Plan de Nación 2010-2022. Y factores asociados al rendimiento académico. https:// postgrado.upnfm.edu.hn/files/VRIP/VRIP\%20POA/POA_2017/SEGUIMIENTO\%20A\%20 INDICADORES\%20EDUCATIVOS\%202016.pdf

Universidad Nacional Autónoma de Honduras (1992). Normas académicas de la educación superior. [Archivo PDF]. https://des.unah.edu.hn/repositorio/normativa

Universidad Nacional Autónoma de Honduras (2018). La Educación Superior de Honduras y sus Lineas Estratégicas de Desarrollo. Universidad Nacional Autónoma de Honduras. https://des. unah.edu.hn/noticias/la-educacion-superior-de-honduras-y-sus-lineas-estrategicas-de-desarrollo

Velásquez de Zapata, C. (2013). Criterios e Indicadores para Evaluar la Calidad de la Educación en Instituciones de Educación Superior. [Archivo PDF]. http://www.ucv.ve/fileadmin/user_upload/ vrac/documentos/Curricular_Documentos/Evento/Ponencias_6/velasquez_de_zapata_carmen.pdf

Zandomeni, N. y Canale, S. (2010). Las trayectorias académicas como objeto de investigación en las instituciones de educación superior. Ciencias Económicas, 2(8), 59-65. https://dialnet.unirioja. es/servlet/articulo? codigo $=5523020$ 